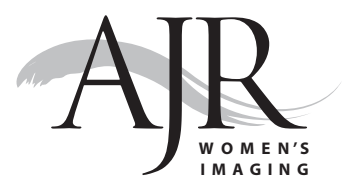

Mijung Jang ${ }^{1}$

Nariya $\mathrm{Cho}^{1}$

Woo Kyung Moon ${ }^{1}$

Jeong Seon Park ${ }^{2}$

Min Hyun Seong ${ }^{1}$

In Ae Park ${ }^{3}$

Keywords: biopsy, breast, breast neoplasms, diagnosis, sonography, technology

\section{DOI:10.2214/AJR.07.3643}

Received January 8, 2008; accepted after revision May 23, 2008.

Supported by grant A062260 from the Innovative Research Institute for Cell Therapy, Republic of Korea.

'Department of Radiology, Clinical Research Institute, and Institute of Radiation Medicine, Seoul National University Hospital, Seoul National University Medical Research Center, 28, Yongon-dong, Chongno-gu, Seoul, 100-744, Korea. Address correspondence to N. Cho (nariya@radiol.snu.ac.kr).

${ }^{2}$ Department of Radiology, College of Medicine, Hanyang University, Seongdong-gu, Seoul, Korea.

${ }^{3}$ Department of Pathology, Seoul National University Hospital, Seoul, Korea.

AJR2008; 191:1347-1351

0361-803X/08/1915-1347

(c) American Roentgen Ray Society

\title{
Underestimation of Atypical Ductal Hyperplasia at Sonographically Guided Core Biopsy of the Breast
}

OBJECTIVE. The purpose of this study was to determine the rate of underestimation of atypical ductal hyperplasia $(\mathrm{ADH})$ at sonographically guided core biopsy of the breast and to identify the factors involved.

MATERIALS AND METHODS. We retrospectively reviewed 3,563 lesions consecutively evaluated with sonographically guided core biopsy between January 2002 and June 2006. Histologic analysis yielded ADH in 60 of the 3,563 lesions (1.7\%). The rate of underestimation of ADH was determined by dividing the number of lesions that proved to be carcinoma at surgical excision by 44 , the total number of lesions evaluated with excisional biopsy. Clinical, sonographic, and core biopsy features were analyzed to identify factors that affect the rate of underestimation of $\mathrm{ADH}$.

RESULTS. The rate of underestimation of ADH was found to be $48 \%$ ( 21 of 44 lesions). Underestimation of ADH was significantly less frequent for lesions evaluated with 11-gauge vacuum-assisted biopsy than for lesions evaluated with 14-gauge automated gun biopsy (22\% [four of 18 lesions] vs $65 \%$ [17 of 26 lesions], $p=0.012$ ). The other clinical, sonographic, and biopsy features examined did not affect the rate of underestimation of ADH.

CONCLUSION. For sonographically guided core biopsy of the breast, the rate of underestimation of ADH was $48 \%$. This rate was lower for lesions evaluated with 11-gauge vacuum-assisted biopsy ( $22 \%$ ) than for those evaluated with 14-gauge automated gun biopsy $(65 \%)$. This finding was particularly true of smaller lesions $(\leq 2.0 \mathrm{~cm})$ and for lesions of the mass-only type.

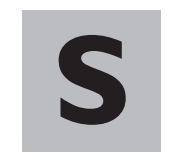

onographically guided percutaneous biopsy of the breast is an established alternative to surgical excision. The advantages of sonography as a guidance technique include lack of ionizing radiation, use of nondedicated equipment, and real-time visualization of the biopsy needle. For lesions amenable to stereotactic and sonographically guided biopsy, sonographically guided biopsy is preferable in terms of patient comfort, procedure time, and cost [1-3].

Atypical ductal hyperplasia $(\mathrm{ADH})$ is the most common high-risk lesion of the breast. It is defined as a ductal hyperplastic lesion that has cellular atypia and structural rigidity suggesting ductal carcinoma in situ (DCIS) but involving only one duct profile or an area less than $2 \mathrm{~mm}$ in diameter [4]. Pathologists have considerable interobserver variability in terms of determining whether a lesion is ADH or DCIS [5], and ADH lesions frequently coexist with DCIS or invasive carcinoma [6]. For these reasons, lesions determined to be $\mathrm{ADH}$ at core needle biopsy often are found to be malignant tumors at subsequent surgery [7, 8]. Reports [9-16] indicate a $20-56 \%$ rate of underestimation of ADH at stereotactic 14-gauge automated core biopsy and a $11-27 \%$ rate at stereotactic 11-gauge vacuum-assisted biopsy. Because most lesions containing ADH have calcifications, underestimation at percutaneous biopsy is most common for calcific lesions examined with stereotactic biopsy [17]. Few reports have addressed the rate of underestimation of $\mathrm{ADH}$ and factors affecting this rate at sonographically guided core biopsy [18-21]. The purpose of this study was to determine the rate of underestimation of $\mathrm{ADH}$ at sonographically guided core biopsy of the breast and to identify the factors involved. 
Jang et al.

\section{Materials and Methods \\ Patients and Lesions}

We retrospectively evaluated the results of sonographically guided core needle biopsy of 3,563 consecutively detected breast lesions in 3,377 consecutively registered patients (mean age, 45.2 years; range, 17-87 years) who were examined between January 2002 and June 2006 at our institution. Of these 3,377 women, 3,202 underwent biopsy for one lesion, 164 women for two separate lesions, and 11 for three separate lesions. Referring physicians reported that 655 of the 3,563 lesions (18.4\%) were palpable. Medical records were reviewed to obtain patient ages, personal medical histories, and family histories of breast cancer.

Sonographically guided percutaneous biopsy yielded benign histologic findings on 2,534 of the 3,563 lesions $(71 \%)$, high-risk findings on 252 lesions (7\%), and malignant findings on 777 lesions (22\%). Biopsy of 60 of the 3,563 lesions (1.7\%) yielded a finding of ADH. Among the 60 women with these lesions, seven women were lost to follow-up, and nine women underwent one or two follow-up imaging examinations 6-32 months after detection without undergoing surgery. No malignancy was found in the follow-up group. Forty-four women with 44 lesions (26 who underwent 14-gauge automated gun biopsy, 18 who underwent 11-gauge vacuum-assisted biopsy) who underwent surgical excision constituted the study group. This study was approved by the institutional review board, which waived the requirement for informed consent.

\section{Biopsy Procedure}

All biopsies were guided by high-resolution sonography performed with a unit equipped with a 10- or 12-MHz linear transducer (Voluson 730, GE-Kretz; HDI 5000, Advanced Technology Laboratories). Patients were in the supine or oblique supine position. A 14-gauge automated gun (Pro-Mag 2.2, Manan Medical Products) was used on 2,463 lesions (69\%) and an 11-gauge vacuum-assisted device (Mammotome, Ethicon Endo-Surgery) on 1,100 lesions (31\%) (Table 1).

Radiologists performing biopsies were primarily responsible for choosing the biopsy device used. Automated gun biopsy was preferred for multifocal lesions and for lesions in the subareolar or axillary area. The 11-gauge vacuum-assisted device was preferred for intraductal lesions and solid nodules smaller than $10 \mathrm{~mm}$ in diameter. When automated gun biopsy was used, needle insertion was immediately proximal to the lesion. After the lesion capsule was pierced, the gun was fired into the lesion for automatic sampling of a small core. After the device was fired, longitudinal

TABLE I: Comparison of I4-Gauge Automated Gun and II-Gauge Vacuum-Assisted Biopsies

\begin{tabular}{l|c|c|c}
\hline \multicolumn{1}{c|}{ Variable } & $\begin{array}{c}\text { 14-Gauge Automated } \\
\text { Gun }(n=2,463)\end{array}$ & $\begin{array}{c}\text { 11-Gauge Vacuum-Assisted } \\
\text { Device }(n=1,100)\end{array}$ & $p$ \\
\hline Histologic diagnosis & 595 & 182 & \\
Malignant lesion & 138 & 114 & \\
High-risk lesion & 1,730 & 804 & \\
Benign lesion & $28(1.1)$ & $32(2.9)$ & $<0.001$ \\
Case inclusion & 26 & 18 & \\
ADH lesion & 17 & 4 & 0.012 \\
ADH lesions managed with surgery & $17 / 26(65)$ & $4 / 18(22)$ & \\
ADH with subsequent malignancy found & & & \\
Rate of underestimation of ADH &
\end{tabular}

Note-Values in parentheses are percentages. ADH = atypical ductal hyperplasia.

and transverse images of needles located in the center of the mass were obtained. Our protocol required that at least four nonfragmented samples be obtained with the automated gun biopsy. When a vacuum-assisted biopsy device was used, a small skin incision was made, and the needle was inserted either through or directly subjacent to the lesion. The needle was manually rotated in a circumferential clockwise manner for sampling of both the target lesion and adjacent tissues. Our protocol required removal of all evidence of the target lesion during vacuum-assisted biopsy.

An average of five core samples (range, three to eight) was obtained with the 14-gauge automated gun and 14 (range, seven to 33) with the 11-gauge vacuum-assisted device. Approximately $20 \%$ of biopsies were performed by one of two attending radiologists with 4-7 years of experience in breast sonography and intervention. The other biopsies were performed by one of four fellows, who performed their first five to 10 biopsies under the supervision of an attending radiologist.

\section{Assessment of Variables}

Lesion variables, including size (maximal diameter on sonograms), type (mass with calcifications or mass only according to mammographic findings), and BI-RADS final assessment category, were determined by two radiologists in consensus before biopsy. According to the probability of malignancy, subcategories of category 4 were described as $3-10 \%$ in 4 a (low level of suspicion), $11-50 \%$ in $4 \mathrm{~b}$ (intermediate suspicion), and $51-94 \%$ in $4 \mathrm{c}$ (moderate suspicion). Biopsy variables including the biopsy method used and number of core specimens retrieved were recorded during biopsy procedures. Histologic results of core biopsy were compared with those obtained during subsequent surgery. Coexistent pathologic lesions and the presence of a second high-risk lesion associated with $\mathrm{ADH}$ were evaluated by review of histology reports at final excision.

\section{Postbiopsy Management}

Patients with a biopsy result of malignancy (invasive ductal carcinoma, DCIS, invasive lobular carcinoma, or malignant phyllodes tumor) underwent surgical excision. Surgical excision was recommended for patients with a high-risk lesion (ADH, atypical papillary tumor, radial scar, benign phyllodes tumor, atypical lobular hyperplasia, lobular carcinoma in situ, or a mucocele-like tumor). When a lesion with a benign pathologic result (all lesions not malignant or high-risk lesions) was determined to have concordant imaging findings, 6-, 12-, and 24-month follow-up imaging examinations were recommended. If a lesion with a benign pathologic result had a highly suspicious sonographic finding, surgical excision was recommended despite the benign pathologic result.

\section{Data Analysis}

Underestimation of ADH was defined as having occurred when the finding of ADH at core biopsy was changed to carcinoma (DCIS or invasive ductal carcinoma) at surgery. The rate of underestimation of $\mathrm{ADH}$ was determined by dividing the number of lesions proved DCIS or invasive carcinoma at excision by the total number of lesions for which excisional biopsy was performed. We also evaluated whether underestimation of ADH was affected by a family or personal history of breast cancer, lesion size, lesion type, final assessment category, biopsy method, number of core specimens, and the presence of a high-risk lesion in the surgical specimen. To identify factors that affected underestimation of $\mathrm{ADH}$, we compared the rates of underestimation of lesions with and without factors related to lesion characteristics. Analysis was performed with the chi-square test, 


\section{Sonographic Breast Biopsy}

Fisher's exact test, and Student's $t$ test in the SPSS program (version 10.0 for Microsoft Windows, SPSS). Values of $p<0.05$ were considered to indicate a significant difference.

\section{Results}

The prevalence of ADH at sonographically guided biopsy was $1.7 \%$ (60 of 3,563 lesions). Of the 60 lesions, 28 were detected with 14-gauge automated gun biopsy (1.1\% of 2,463 lesions biopsied with this technique) and 32 with 11-gauge vacuum-assisted biopsy $(2.9 \%$ of 1,100 biopsied with this technique) ( $p<0.001)$. Forty-four lesions were surgically excised, and 21 of these lesions proved to be carcinoma (12 DCIS, nine invasive ductal carcinomas) (Table 1), an ADH underestimation rate of $48 \%$ (21 of 44 lesions).

Table 2 compares the accurate diagnoses $(n=23)$ and underestimations $(n=21)$ according to patient, lesion, and biopsy variables. Women in the accurate diagnosis group were younger than those in the underestimation group (mean age, 46 vs 52 years; $p=0.016$ ). Seventeen of the $26 \mathrm{ADH}$ lesions $(65 \%)$ found with 14-gauge automated gun biopsy were upgraded to carcinoma (10 DCIS, seven invasive ductal carcinomas), and four of the $18 \mathrm{ADH}$ lesions (22\%) found with 11-gauge vacuum-assisted biopsy were upgraded to carcinoma (two DCIS, two invasive ductal carcinomas). The underestimation rate for 11-gauge vacuum-assisted biopsy (22\%) was significantly lower than that for 14-gauge automated gun biopsy $(65 \%)(p=0.012)$.

No difference was found between the accurate diagnosis and underestimation groups in terms of family or personal history of breast cancer $(p=1.0)$, lesion size $(p=0.272)$, lesion type $(p=0.761)$, BI-RADS category $(p=0.559)$, number of core specimens $(p=0.108)$, or presence of combined high-risk lesions $(p=0.526)$. The accurate diagnosis group had three papillary lesions, one radial scar, and two mucocelelike lesions; the underestimation group had four papillary lesions, one radial scar, and one lobular carcinoma in situ.

The underestimation rates for 14-gauge automated gun biopsy and 11-gauge vacuumassisted biopsy were compared with respect to lesion characteristics (Table 3). The rate of underestimation of $\mathrm{ADH}$ at 14-gauge automated gun biopsy was higher than that of 11-gauge vacuum-assisted biopsy for smaller $(\leq 2.0 \mathrm{~cm})$ lesions $(61 \%$ [14 of 23 lesions] versus $8 \%$ [one of 12], $p=0.004)$ ] and mass-only lesions (67\% [14 of 21] versus $0 \%$ (zero of seven), $p=0.009$ ). No difference was observed between the underestimation rates of the two biopsy methods for larger $(\geq 2.1 \mathrm{~cm})$ lesions $(p=0.464)$ or masses with calcification $(p=0.734)$. There also were no differences related to BI-RADS category.

\section{Discussion}

In this study, the rate of underestimation of $\mathrm{ADH}$ at sonographically guided core biopsy was found to be $48 \%$ ( 21 of 44 lesions) among 3,563 consecutive sonographically guided core needle biopsies of the breast. Our rate of underestimation of ADH with sonographically guided biopsy concurs with those in previous reports of stereotactic

\section{TABLE 2: Comparison of Accurately Diagnosed and Underestimated Lesions}

\begin{tabular}{|c|c|c|c|}
\hline Feature & $\begin{array}{l}\text { Accurately Diagnosed } \\
\qquad(n=23)\end{array}$ & $\begin{array}{l}\text { Underestimated } \\
\qquad(n=21)\end{array}$ & $p$ \\
\hline Mean \pm SD patient age (y) & $46 \pm 9.4$ & $52 \pm 8.3$ & 0.016 \\
\hline Family or personal history of breast cancer & & & 1.0 \\
\hline Present & 4 & 4 & \\
\hline Absent & 19 & 17 & \\
\hline Mean \pm SD lesion size $(\mathrm{cm})$ & $1.3 \pm 0.7$ & $1.6 \pm 1.0$ & 0.394 \\
\hline Lesion size & & & 0.272 \\
\hline$\leq 2.0$ & 20 & 15 & \\
\hline$\geq 2.1$ & 3 & 6 & \\
\hline Lesion type & & & 0.761 \\
\hline Mass with calcifications & 9 & 7 & \\
\hline Mass only & 14 & 14 & \\
\hline BI-RADS category & & & 0.559 \\
\hline 3 & 1 & 1 & \\
\hline $4 a$ & 14 & 10 & \\
\hline $4 b$ & 6 & 7 & \\
\hline $4 c$ & 2 & 1 & \\
\hline 5 & 0 & 2 & \\
\hline Biopsy method & & & 0.012 \\
\hline 14-gauge automated gun & 9 & 17 & \\
\hline 11-gauge vacuum assisted & 14 & 4 & \\
\hline No. of specimens & & & 0.108 \\
\hline$\leq 5$ & 5 & 11 & \\
\hline $6-10$ & 11 & 6 & \\
\hline$\geq 11$ & 7 & 4 & \\
\hline $\begin{array}{l}\text { Second high-risk lesion with ADH at core } \\
\text { biopsy }\end{array}$ & & & 0.526 \\
\hline Present & 6 & 6 & \\
\hline Absent & 17 & 15 & \\
\hline
\end{tabular}

Note-ADH = atypical ductal hyperplasia. biopsy: a 20-56\% rate of underestimation of ADH with 14-gauge automated gun biopsy and an 11-27\% rate with 11-gauge vacuumassisted biopsy [9-12]. However, the clinical significance of underestimation of ADH with sonographically guided biopsy is much lower than that of stereotactic biopsy. Whereas a 4-7\% prevalence of $\mathrm{ADH}$ at stereotactic biopsy has been reported [9-16], in our study, the prevalence of ADH was only $1.7 \%$ (60 of 3,563 lesions) with sonographically guided biopsy. Our finding concurs with the ADH prevalences of $0.4-2.5 \%$ previously reported for sonographically guided biopsy [18-21]. Prevalence of ADH determined with percutaneous biopsy depends on lesion type; that is, the prevalence of $\mathrm{ADH}$ increases 


\section{Jang et al.}

TABLE 3: Rates of Underestimation of Atypical Ductal Hyperplasia for I4-Gauge Automated Gun and II-Gauge Vacuum-Assisted Biopsies with Respect to Lesion Characteristics

\begin{tabular}{l|c|c|c|c}
\hline \multicolumn{1}{c|}{ Feature } & $\begin{array}{c}14-\text { Gauge } \\
\text { Automated Gun } \\
(n=26)\end{array}$ & $\begin{array}{c}11 \text {-Gauge Vacuum- } \\
\text { Assisted Device } \\
(n=18)\end{array}$ & Total & $p$ \\
\hline Lesion size (cm) & $14 / 23(61)$ & $1 / 12(8)$ & $15 / 35(43)$ & 0.004 \\
$\leq 2.0$ & $3 / 3(100)$ & $3 / 6(50)$ & $6 / 9(67)$ & 0.464 \\
$\geq 2.1$ & $3 / 5(60)$ & $4 / 11(36)$ & $7 / 16(44)$ & 0.734 \\
Lesion type & $14 / 21(67)$ & $0 / 7(0)$ & $14 / 28(50)$ & 0.009 \\
Mass with & & 0 & & \\
calcifications & $1 / 2(50)$ & 0 & $1 / 2(50)$ & \\
Mass only & $7 / 13(54)$ & $3 / 11(27)$ & $10 / 24(42)$ & 0.240 \\
BI-RADS category & $6 / 8(75)$ & $1 / 5(20)$ & $7 / 13(54)$ & 0.103 \\
3 & $1 / 1(100)$ & $0 / 2(0)$ & $1 / 3(33)$ & \\
$4 a$ & $2 / 2(100)$ & 0 & $2 / 2(100)$ & \\
$4 b$ & & 0 & \\
$4 c$ & & & \\
5 & & & & \\
\hline
\end{tabular}

Note-Values in parentheses are percentages.

when more lesions with calcifications are included [16]. However, lesions manifesting as calcifications alone are rarely encountered at sonography; thus the prevalence of $\mathrm{ADH}$ is minimal as determined with sonographically guided biopsy.

The outcome of percutaneous biopsy of the breast depends mainly on lesion type and the amount of sample obtained. In this study, use of an 11-gauge vacuum-assisted device was associated with a lower rate of underestimation of ADH compared with 14-gauge core biopsy. This finding was notable for small lesions $(\leq 2.0 \mathrm{~cm})(8 \%$ [one of 12 lesions] vs $61 \%$ [14 of 23], $p=$ $0.004)$ and for mass-only lesions $(0 \%$ [zero of seven lesions] vs 67\% [14 of 21], $p=$ 0.009). Many studies [9-12] of stereotactic biopsy have shown that 11-gauge vacuumassisted devices remove larger amounts of tissue than do 14-gauge core biopsy devices and that use of 11-gauge devices is associated with a lower rate of underestimation of ADH. These findings may be applicable to sonographically guided biopsy. Calcific lesions, in which ADH is commonly found, have a heterogeneous and discontinuous distribution; thus larger sample volumes are needed than for mass lesions [17, 22]. In one stereotactic biopsy series [23], the rate of underestimation of $\mathrm{ADH}$ was higher for masses than for microcalcifications. Our results, however, suggest that use of 11-gauge vacuum-assisted biopsy reduces the rate of underestimation of masses alone but not of masses with calcifications at sonographically guided biopsy. Because calcifications are barely visible at sonography, calcifications remaining after biopsy were thought be the cause of underestimation.

The partial volume averaging effect is a well-known cause of inadequate targeting during sonographically guided biopsy, notably of small lesions. With vacuum-assisted devices, samples can be contiguous and larger than with the 14-gauge gun, and all evidence of lesions can be removed. These features overcome the partial volume averaging effect of small lesions and minimize the residual lesions that cause histologic underestimation. Sonographically guided vacuum-assisted removal of all sonographic evidence of a lesion was associated with a $0 \%$ (zero of 29 lesions) rate of underestimation of $\mathrm{ADH}$ in one study [19]. Even complete removal of all mammographic evidence at stereotactic 11-gauge vacuum-assisted biopsy, however, revealed residual lesion during surgical excision [24] and had an 8\% (three of 36 lesions) rate of underestimation of ADH [16].

Although reduced underestimation with use of an 11-gauge vacuum-assisted device is explained by larger sample volumes, there is a tendency to obtain more cores for larger lesions or lesions that are difficult to target. Thus the number of specimens obtained appears not to be correlated with a lower rate of underestimation (Table 2). These results are similar to those of a previous study [16], in which the investigators found that specimen numbers per lesion did not correlate with underestimation but that complete lesion removal did correlate with degree of underestimation. These findings indicate that targeting precision is more important than sample numbers. Further studies with more cases are needed to determine whether complete lesion removal at sonographically guided 11-gauge vacuum-assisted biopsy can reduce the rate of underestimation of $\mathrm{ADH}$.

We also evaluated whether finding a second high-risk lesion at core biopsy was related to rate of underestimation of $\mathrm{ADH}$, hoping to find a histologic feature at core biopsy that could serve as a marker for high risk. We found no difference between the accurate diagnosis and underestimation groups in terms of presence of a second highrisk lesion at core biopsy (Table 2).

Our study had several limitations. First, biopsies were performed by one of six radiologists with different levels of experience. Although the first five to 10 biopsies by fellows were performed under the supervision of an attending radiologist, the training process, especially concerning accurate targeting, might have been incomplete. Second, decisions concerning the biopsy device used were not randomized. A 14-gauge automated gun was preferred for multifocal lesions and for lesions in the subareolar or axillary area, and an 11-gauge vacuum-assisted device was preferred for intraductal lesions and solid nodules smaller than $10 \mathrm{~mm}$, for which the potential benefits of use of the vacuum-assisted device had already been suggested. Third, our biopsy protocols involved removal of all evidence of lesions targeted during 11-gauge vacuum-assisted biopsy and acquisition of at least four nonfragmented samples during 14-gauge automated gun biopsy, and these factors might have affected the rate of underestimation of ADH.

For sonographically guided core biopsy of the breast, the rate of underestimation of $\mathrm{ADH}$ was found to be $48 \%$. This rate was lower for 11-gauge vacuum-assisted biopsy $(22 \%)$ than for 14-gauge automated gun biopsy (65\%), notably for lesions $20 \mathrm{~mm}$ or smaller and for lesions of the mass-only type. These findings, however, do not mean that we prefer vacuum-assisted biopsy to automated gun biopsy for all sonographic 


\section{Sonographic Breast Biopsy}

lesions in clinical practice. The decision about which biopsy method to use also depends on procedure-related complications, cost-effectiveness, and time.

\section{References}

1. Parker SH, Jobe WE, Dennis MA, et al. US-guided automated large-core breast biopsy. Radiology 1993; 187:507-511

2. Liberman L, Feng TL, Dershaw DD, Morris EA, Abramson AF. Ultrasound-guided core breast biopsy: utility and cost-effectiveness. Radiology 1998; 208:717-723

3. Smith DN, Darling ML, Meyer JE, et al. The utility of ultrasonographically guided large-core needle biopsy: results from 500 consecutive breast biopsies. J Ultrasound Med 2001; 20:43-49

4. Tavassoli FA, Norris HJ. A comparison of the results of long-term follow-up for atypical intraductal hyperplasia and intraductal hyperplasia of the breast. Cancer 1990; 65:518-529

5. Schnitt SJ, Connolly JL, Tavassoli FA, et al. Interobserver reproducibility in the diagnosis of ductal proliferative breast lesions using standardized criteria. Am J Surg Pathol 1992; 16:1133-1143

6. Moore MM, Hargett CW III, Hanks JB, et al. Association of breast cancer with the finding of atypical ductal hyperplasia at core breast biopsy. Ann Surg 1997; 225:726-731

7. Sewell CW. Pathology of high-risk breast lesions and ductal carcinoma in situ. Radiol Clin North Am 2004; 42:821-830

8. Berg WA. Image-guided breast biopsy and management of high-risk lesions. Radiol Clin North
Am 2004; 42:935-946

9. Jackman RJ, Burbank F, Parker SH, et al. Atypical ductal hyperplasia diagnosed at stereotactic breast biopsy: improved reliability with 14-gauge, directional, vacuum-assisted biopsy. Radiology 1997; 204:485-488

10. Meyer JE, Smith DN, Lester SC, et al. Large-core needle biopsy of nonpalpable breast lesions. JAMA 1999; 281:1638-1641

11. Darling ML, Smith DN, Lester SC, et al. Atypical ductal hyperplasia and ductal carcinoma in situ as revealed by large-core needle breast biopsy: results of surgical excision. AJR 2000; 175:1341-1346

12. Philpotts LE, Shaheen NA, Carter D, Lange RC, Lee $\mathrm{CH}$. Comparison of rebiopsy rates after stereotactic core needle biopsy of the breast with 11-gauge vacuum suction probe versus 14-gauge needle and automatic gun. AJR 1999; 172:683-687

13. Kettritz U, Rotter K, Schreer I, et al. Stereotactic vacuum-assisted breast biopsy in 2874 patients: a multicenter study. Cancer 2004; 100:245-251

14. Winchester DJ, Bernstein JR, Jeske JM, et al. Upstaging of atypical ductal hyperplasia after vacuum-assisted 11-gauge stereotactic core needle biopsy. Arch Surg 2003; 138:619-622

15. Pfarl G, Helbich TH, Riedl CC, et al. Stereotactic 11-gauge vacuum-assisted breast biopsy: a validation study. AJR 2002; 179:1503-1507

16. Jackman RJ, Birdwell RL, Ikeda DM. Atypical ductal hyperplasia: can some lesions be defined as probably benign after stereotactic 11-gauge vacuum assisted biopsy, eliminating the recommendation for surgical excision? Radiology 2002;
224:548-554

17. Liberman L, Dershaw DD, Glassman JR, et al. Analysis of cancers not diagnosed at stereotactic core breast biopsy. Radiology 1997; 203:151-157

18. Philpotts LE, Hooley RJ, Lee CH. Comparison of automated versus vacuum-assisted biopsy methods for sonographically guided core biopsy of the breast. AJR 2003; 180:347-351

19. Grady I, Gorsuch H, Wilburn-Bailey S. Ultrasound-guided, vacuum-assisted, percutaneous excision of breast lesions: an accurate technique in the diagnosis of atypical ductal hyperplasia. $J$ Am Coll Surg 2005; 201:14-17

20. Cho N, Moon WK, Cha JH, et al. Sonographically guided core biopsy of the breast: comparison of 14-gauge automated gun and 11-gauge directional vacuum-assisted biopsy methods. Korean J Radiol 2005; 6:102-109

21. Youk JH, Kim EK, Kim MJ, Oh KK. Sonographically guided 14-gauge core needle biopsy of breast masses: a review of 2,420 cases with long-term follow-up. AJR 2008; 190:202-207

22. Liberman L. Percutaneous image-guided core breast biopsy. Radiol Clin North Am 2002; 40:483-500

23. Jackman RJ, Burbank F, Parker SH, et al. Stereotactic breast biopsy of nonpalpable lesions: determinants of ductal carcinoma in situ underestimation rates. Radiology 2001; 218:497-502

24. Liberman L, Kaplan JB, Morris EA, Abramson AF, Menell JH, Dershaw DD. To excise or to sample the mammographic target: what is the goal of stereotactic 11-gauge vacuum-assisted breast biopsy? AJR 2002; 179:679-683 